\title{
Mecanismos de acción de la anticoncepción hormonal de emergencia: efectos del levonorgestrel anteriores y posteriores a la fecundación
}

\author{
Marta Durand, MD, (1) Fernando Larrea, MD, (1) Raffaela Schiavon, MD.(2)
}

\section{Durand M, Larrea F, Schiavon R. Mecanismos de acción de la anticoncepción hormonal de emergencia: efectos del levonorgestrel anteriores y posteriores a la fecundación. Salud Publica Mex 2009;5 I:255-26I.}

\section{Resumen}

El mecanismo de acción del levonorgestrel (LNG) como anticonceptivo de emergencia ( $A E)$ es aún controvertido. Para quienes consideran que el embarazo inicia antes de la implantación, todo compuesto capaz de interferir con etapas posteriores a la fecundación y anteriores a la implantación se considera abortivo. Investigaciones previas sugieren que en seres humanos este método actúa también después de la fecundación. Sin embargo, en la actualidad existe sólida evidencia que demuestra que los efectos anteriores a la fecundación son en realidad los que explican la acción anticonceptiva del LNG. En este artículo se revisa la evidencia acumulada sobre los mecanismos de acción propuestos. Los consensos derivados de la información disponible establecen que los mecanismos prefecundación (inhibición o retardo de la ovulación) son los que explican la efectividad anticonceptiva de los AE de progestina sola.

Palabras clave: anticoncepción de emergencia; levonorgestrel; post-coital; fecundación; implantación
Durand M, Larrea F, Schiavon R.

Mechanisms of action of emergency contraception: pre and post-fertilization effects

of levonorgestrel.

Salud Publica Mex 2009;5 1:255-26I.

\begin{abstract}
There is still controversy regarding the mechanism of action of levonorgestrel (LNG) for emergency contraception (EC). For those who state that pregnancy starts prior to implantation, any compound able to interfere with post-fertilization and pre-implantation stages, should be considered as abortifacient. Previous research suggests that $\mathrm{EC}$ in humans acts predominantly after fertilization. Current evidence with LNG-only EC supports a pre-fertilization mechanisms to explain its action. There are many potential mechanisms of action, which could vary pending on the day during the fertilization window of the ovarian cycle at which the contraceptive is given. This paper reviews the evidence for each potential mechanism of action. According to the most recently statements, it is concluded that the primary and possible the only mechanism of action of LNG-only EC is preventing or delaying ovulation.
\end{abstract}

Key words: emergency contraception; levonorgestrel; postcoital; fertilization; implantation

(I) Departamento de Biología de la Reproducción, Instituto Nacional de Ciencias Médicas y Nutrición Salvador Zubirán. México DF.

(2) Ipas México. México DF.

Fecha de recibido: 30 de octubre de 2008 - Fecha de aprobado: 10 de febrero de 2009 Solicitud de sobretiros: Dra. Raffaela Schiavon. Ipas México. Concepción Béistegui 106, col. Del Valle. 03100, México DF. Correo electrónico: schiavonr@ipas.org 
L a incorporación de la anticoncepción de emergencia (AE) en los servicios de salud, en particular en los países de la región latinoamericana, ha generado una polarización de opiniones, en particular en relación con sus potenciales efectos antiimplantatorios. ${ }^{1,2}$ Hoy en día existe suficiente evidencia científica acerca del mecanismo de acción del LNG en la AE, la cual se basa en modelos estadísticos y estudios efectuados en seres humanos y diferentes especies animales. Con base en el análisis de algunos datos estadísticos indirectos vinculados con la efectividad de la $\mathrm{AE}$ se ha sugerido la existencia de otros mecanismos, además de la inhibición de la ovulación. ${ }^{3,4}$ El objetivo de la presente revisión fue examinar los protocolos publicados sobre el mecanismo de acción de la AE y ponderar la contribución de sus efectos anteriores y posteriores a la fecundación. Los efectos prefecundación se definen como aquellos que reducen la probabilidad de ovulación o fecundación del ovocito y los posfecundación se refieren a todos los observados después de la fecundación, incluidos los relativos a la receptividad endometrial del concepto.

\section{Mecanismos de acción propuestos}

Se han propuesto diferentes mecanismos de acción, todos teóricamente posibles para un anticonceptivo poscoital y con una ventana de eficacia promedio de $72 \mathrm{~h}$. Éstos incluyen aquellos que interfieren con los procesos de desarrollo folicular, ovulación, transporte y capacitación espermática, fecundación, desarrollo y transporte del cigoto, receptividad uterina y los procesos de adhesión endometrial y función del cuerpo lúteo en etapas tempranas del embarazo. ${ }^{1}$ La administración preovulatoria de LNG en el esquema utilizado en la $\mathrm{AE}$ interfiere con el desarrollo folicular y por tanto con el proceso ovulatorio. ${ }^{5-7}$ Este mecanismo de acción explica con claridad su efectividad anticonceptiva en la fase preovulatoria. En las décadas de 1970 y 1980, algunos estudios demostraron alteraciones histológicas o bioquímicas en el endometrio después de la administración del esquema combinado de emergencia, ${ }^{8-10}$ lo que sugirió sus efectos endometriales al impedir la receptividad uterina y por consiguiente la implantación del concepto. No obstante, estas investigaciones no consideraron las fases del ciclo tanto de la administración del medicamento como de la obtención del espécimen de tejido endometrial. La inclusión de estas variables en estudios recientes mostraron la ausencia de efectos endometriales con la formulación combinada y la progestina sola., ${ }^{5,11}-13$

\section{Efectos sobre la ovulación}

La posibilidad de que la $\mathrm{AE}$ interfiera con la ovulación se ha explorado desde los primeros estudios clínicos mediante la combinación de estrógenos y progestina. Estos estudios mostraron que la inhibición de la ovulación dependió de la fase del ciclo en que se instituyó el tratamiento. Estudios posteriores con LNG confirmaron esta observación..$^{5-7,16,17}$ Por ejemplo, la administración de LNG el día de la máxima secreción de hormona luteinizante $(\mathrm{LH}+0)$ inhibió la rotura folicular en 5 de 12 casos en el estudio de Hapangama y colaboradores ${ }^{16}$ y en ninguno en el estudio de Durand y colegas. ${ }^{5}$ En el estudio de Durand y colaboradores, la administración de LNG en la fase folicular (LH-4) indujo la inhibición o retraso de la ovulación en 80 y 20\%, respectivamente, con resultados similares en los estudios de Marions y colegas. ${ }^{7,17}$ Croxatto y colaboradores, ${ }^{18,19}$ en dos estudios independientes, aleatorizados y controlados con placebo, evaluaron los efectos del LNG solo o combinado con etinilestradiol según fuera el diámetro folicular. Los resultados demostraron y confirmaron la observación previa de Durand y colaboradores ${ }^{5}$ acerca del efecto inhibitorio de la ovulación dependiente del diámetro folicular y la fase del ciclo al momento del tratamiento.

En suma, la administración de LNG solo o combinado durante las fases preovulatorias (folicular) tiene diferentes efectos sobre la función ovárica, que dependen del grado de desarrollo y crecimiento folicular. En la mayoría de los casos, los efectos inhibitorios se observan cuando el folículo no alcanza todavía un desarrollo completo $(<18 \mathrm{~mm})$; empero, en algunos casos estos efectos se acompañan de cambios en la disminución de la funcionalidad del cuerpo lúteo. $5,18,19$

\section{Efectos sobre la migración espermática}

La mayor parte de los estudios in vitro diseñados para evaluar los efectos del LNG sobre el espermatozoide no ha mostrado alteraciones significativas sobre la funcionalidad de los gametos masculinos. ${ }^{20-22}$ Sin embargo, de acuerdo con dos protocolos, el LNG ha mostrado cambios en las propiedades físico-químicas del moco cervical y afectación de la penetración espermática. ${ }^{23,24}$ Kesserü y colaboradore ${ }^{25}$ informaron que la administración de $0.4 \mathrm{mg}$ de LNG, 3 a 10 horas después del coito, produjo la disminución del número de espermatozoides recuperados de la cavidad uterina, lo cual modificó en especial la fase de migración espermática 
sostenida. Bajo condiciones fisiológicas, los espermatozoides localizados en las criptas del cuello uterino conservan su viabilidad y capacidad fecundante hasta por cinco días poscoito. A este respecto, es bien conocido que luego de la fase de migración rápida, durante la cual los espermatozoides llegan a la trompa uterina, existe una fase de migración sostenida en la cual los espermatozoides atraviesan en cohortes sucesivas el reservorio cervical. ${ }^{26}$ Estudios llevados a cabo con estas dos poblaciones de espermatozoides revelan que sólo los contenidos en la fase sostenida tienen la capacidad de interactuar con el ovocito. ${ }^{26}$ Estos resultados sugieren que parte de la acción anticonceptiva del LNG es secundaria a sus efectos sobre las fases de migración espermática y por lo tanto de su capacidad de interactuar con los gametos femeninos.

\section{Efectos sobre el endometrio}

La posibilidad de que la AE en sus diferentes formulaciones inhiba la implantación del óvulo fecundado a través de modificar y alterar el tejido endometrial constituye el punto más controvertido dentro de sus mecanismos de acción. Los primeros artículos que refieren alteraciones endometriales con la AE corresponden a las experiencias iniciales con el uso poscoital del esquema combinado. ${ }^{8-10}$ Sin embargo, dichos estudios muestran serias limitaciones metodológicas, entre ellas la ausencia de controles adecuados, el análisis ciego de los resultados y la obtención inadecuada de las biopsias endometriales. Estudios recientes y con diseños experimentales más apropiados no han podido demostrar efectos de la AE sobre la morfología y otros parámetros bioquímicos del endometrio que expliquen los efectos deletéreos sobre la implantación. 5,27,28 Por ejemplo, el estudio de Durand y colaboradores ${ }^{5}$ en biopsias de endometrios obtenidas de mujeres tratadas con LNG durante las fases preovulatoria y ovulatoria del ciclo menstrual $(\mathrm{LH}+0 \mathrm{y}$ LH+2) no mostró cambios de consideración en los diferentes marcadores histológicos o morfométricos cuando se compararon con los obtenidos en el grupo control. De manera interesante, estos autores, al utilizar este mismo esquema de administración de LNG, tampoco demostraron cambios significativos, respecto del grupo control, sobre los numerosos marcadores bioquímicos de receptividad endometrial estudiados (cuadro I).5,28 Marions y colaboradores ${ }^{7}$ describieron resultados similares, incluidas la presencia y la distribución normales de los pinópodos (importante marcador morfológico de receptividad endometrial) en endometrios de mujeres tratadas con LNG. Ugocsai y colaboradores informaron la pérdida de pinópodos en mujeres tratadas con $\mathrm{LNG}_{;}^{, 29}$ empero, las dosis de LNG fueron 4 a 6 veces superiores

\section{Cuadro I \\ PARÁmetros MORFométricos Y MARCADORES DE RECEPTIVIDAD ENDOMETRIAL EN BIOPSIAS DE ENDOMETRIOS DE MUJERES TRATADAS CON LEVONORGESTREL}

Parámetro morfométrico Control $(n=4 I) \quad$ LNG $(n=24)$ media $\pm D E \quad$ media $\pm D E$

\begin{tabular}{lcc} 
No. de glándulas $/ \mathrm{mm}^{2}$ & $29.3 \pm 6.3$ & $28.5 \pm 3.8$ \\
\hline Porcentaje de edema estromal & $52.7 \pm 15.4$ & $55.9 \pm 12.1$ \\
\hline No. de arterias espirales/campo & $5.6 \pm 3.1$ & $4.5 \pm 2.7$
\end{tabular}

$\begin{array}{ccc}\text { Marcador de receptividad } & \text { Control }(n=4 I) & \text { LNG }(n=24) \\ \text { endometrial (unidades de densidad } & \text { media } \pm E E & \text { media } \pm E E \\ \left.\text { óptical } / 500 \mu^{2}\right) & \end{array}$

$\begin{array}{lll}\text { Integrina } \alpha v-\beta 3 & 15 \pm 5 & 19 \pm 5\end{array}$

\begin{tabular}{lll}
\hline Mucina I & $140 \pm 7$ & $113 \pm 9$
\end{tabular}

\begin{tabular}{lll} 
Calcitonina & $69 \pm 8$ & $47 \pm 6$ \\
\hline
\end{tabular}

Factor inhibidor de la leucemia $\quad 36 \pm 6 \quad 21 \pm 4$

Factor de crecimiento epidermal $25 \pm 4 \quad 21 \pm 4$

unido a la heparina

Factor de crecimiento epidermal $\quad 89 \pm 8 \quad 94 \pm 8$

Gene Hox-II $\quad 67 \pm 10 \quad 56 \pm 8$

LNG: levonorgestrel

a las utilizadas en $\mathrm{AE}$, con imprecisiones en el diseño experimental y los tejidos control empleados. La misma condición ocurre con los hallazgos que publicaron Landgren y colegas ${ }^{30}$ y Kahlenbom y colaboradores; ${ }^{31}$ estos últimos, de manera inexplicable, excluyen de su revisión las publicaciones de Durand y colaboradores ${ }^{5}$ y Marions y colaboradores ${ }^{7}$ (cuadro II).

En resumen, las evidencias obtenidas a partir de estudios con diseños experimentales adecuados, ajustados a las dosis y esquemas utilizados en la $\mathrm{AE}$, indican la ausencia de efectos significativos en el endometrio que apoyen efectos antiimplantatorios derivados de la administración de LNG.

\section{Estudios en modelos animales}

Los estudios realizados en modelos animales permiten examinar los efectos del LNG sobre etapas críticas del proceso reproductivo, lo que no puede realizarse en la mujer por limitaciones éticas y logísticas. No obstante, es evidente el cuidado que debe tenerse al extrapolar los resultados a la especie humana, sobre todo en aquellas especies con mayores diferencias en los patrones endocrinos y reproductivos. Müller y colaboradores ${ }^{32}$ observaron en la rata la inhibición parcial o total de la ovulación con LNG, según fueran el momento del trata- 
Cuadro II

EfECTOS ENDOMETRIALES DE LA ANTICONCEPCIÓN DE EMERGENCIA

Esquema Hallazgos Comentario Referencia

\begin{tabular}{|c|c|c|c|}
\hline Combinado & Retardo de la maduración estromaglandular & Biopsias obtenidas en la menstruación & Yuzpe y col. ${ }^{10}$ \\
\hline Combinado & Asincronía del desarrollo estromaglandular & Biopsias obtenidas en la menstruación & Ling y col. ${ }^{8}$ \\
\hline Combinado & $\begin{array}{l}\text { Asincronía estromaglandular y aumento del edema } \\
\text { estromal }\end{array}$ & Biopsias de la ventana de implantación & Ling $y$ col. ${ }^{14}$ \\
\hline Combinado & $\begin{array}{l}\text { Reducción de los receptores endometriales de } \\
\text { esteroides }\end{array}$ & Biopsias obtenidas $24 \mathrm{~h}$ después de la $\mathrm{AE}$ & Kubba y col. ${ }^{9}$ \\
\hline LNG & Sin efectos en la morfología endometrial & Biopsias de la ventana de implantación & Landgren y col. ${ }^{30}$ \\
\hline Combinado & Expresión normal de integrinas & Biopsias de la ventana de implantación & Taskin y col. ${ }^{12}$ \\
\hline Combinado & $\begin{array}{l}\text { Expresión normal de diversos marcadores de } \\
\text { receptividad }\end{array}$ & Biopsias de la ventana de implantación & Raymond y col. ${ }^{13}$ \\
\hline LNG & Sin efectos en la morfología endometrial & $\begin{array}{l}\text { Biopsias de la ventana de implantación. } \\
\text { Análisis de acuerdo con la fase del ciclo }\end{array}$ & Durand y col. ${ }^{5}$ \\
\hline LNG & $\begin{array}{l}\text { Sin efectos en la morfometría, inmunohistoquímica y } \\
\text { microscopia electrónica del endometrio }\end{array}$ & $\begin{array}{l}\text { Biopsias de la ventana de implantación. } \\
\text { Análisis de acuerdo con la fase del ciclo }\end{array}$ & Marions y col. ${ }^{7}$ \\
\hline LNG & Ausencia de pinópodos & Dosis $4-6$ veces mayores a la $A E$ & Ugocsai y col. ${ }^{29}$ \\
\hline $\begin{array}{l}\text { Combinado y } \\
\text { LNG }\end{array}$ & $\begin{array}{l}\text { Revisión de las publicaciones que sustentan efectos } \\
\text { posfecundación }\end{array}$ & $\begin{array}{l}\text { Excluye los estudios de Durand y col. }{ }^{5} \text { y } \\
\text { Marions y col. }\end{array}$ & Kahlenbom y col. ${ }^{31}$ \\
\hline LNG & $\begin{array}{l}\text { Sin efectos en la morfometría e inmunohistoquímica } \\
\text { del endometrio }\end{array}$ & $\begin{array}{l}\text { Biopsias de la ventana de implantación. } \\
\text { Análisis de acuerdo con la fase del ciclo }\end{array}$ & Durand y col. ${ }^{28}$ \\
\hline LNG & $\begin{array}{l}\text { Estudio de efectividad que sustenta efectos } \\
\text { prefecundación }\end{array}$ & $\begin{array}{l}\text { Evidencia que descarta mecanismos de } \\
\text { acción en fase posovulatoria }\end{array}$ & Novikova y col. ${ }^{37}$ \\
\hline
\end{tabular}

LNG: levonorgestrel

miento y la dosis administrada. De manera interesante, en animales ovulatorios, el LNG no interfirió con los procesos de la fecundación y la implantación. Ortiz y colegas, ${ }^{33}$ en la mona Cebus apella, cuyo ciclo hormonal y reproductivo es parecido al de la especie humana, observaron que la administración posovulatoria de LNG no modificó la tasa de embarazos comparada con lo observado en el grupo no tratado (en ambos casos fue de $54.2 \%$ ), lo cual indicó la ausencia de efectos antiimplantatorios del LNG administrado luego de la fecundación. De manera similar al modelo humano, la capacidad del LNG de suprimir la ovulación en la mona Cebus apella dependió del diámetro folicular. ${ }^{18,33}$ Estos protocolos han permitido sugerir que el efecto anticonceptivo del LNG en la $\mathrm{AE}$ es el resultado de la inhibición de la ovulación y no de sus efectos posfecundación endometrial.

\section{Evidencias estadísticas indirectas que descartan efectos posfecundación}

Existen modelos que toman en consideración el intervalo entre el coito y la administración del LNG para explicar el mecanismo de acción de la AE. La primera evidencia resultó de la observación de que la eficacia es mayor a medida que el intervalo es menor ${ }^{34,35}$ (figura 1) y la segunda de que la falla del método es directamente proporcional al intervalo entre el coito y el tratamiento. Estas evidencias sugieren que los efectos anticonceptivos de este método se localizan antes de la implantación del concepto, ya que este proceso temporalmente se lleva a cabo 8 a 11 días después de la ovulación..$^{36}$ Por otra parte, este intervalo perdería significado si el LNG actuara sobre el endometrio y suprimiera la receptividad uterina, ya que se esperaría que la ventana de efectividad anticonceptiva (72 h) fuera mayor. Otras evidencias indirectas se derivan del análisis de las fallas anticonceptivas en casos de coitos únicos y repetidos. ${ }^{34} \mathrm{~A}$ este respecto, la eficacia resultó ser significativamente menor en los casos de coitos repetidos. Esta observación descarta los efectos sobre la implantación, dado que de lo contrario la eficacia anticonceptiva se mantendría elevada a lo largo de toda la fase posovulatoria al margen del número de coitos. 


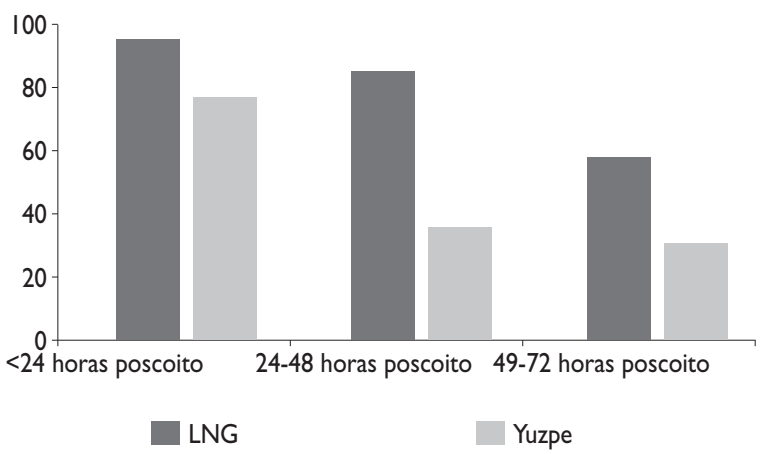

Figura I. Relación entre la efectividad de la antiCONCEPCIÓN DE EMERGENCIA EN SUS DOS MODALIDADES, COMBINADA (YUZPE) O LA PROGESTINA SOLA (LNG), Y EL INTERVALO COITO-TRATAMIENTO. EL CÁLCULO DE LOS PORCENTAJES (EFECTIVIDAD) INCLUYE LA PROBABILIDAD ESPERADA DE EMBARAZO DE ACUERDO A WILCOX Y COLABORADORES ${ }^{38}$

\section{Recientes evidencias directas que descartan efectos posfecundación}

Novikova y colegas informaron la ausencia de efectos posovulatorios del LNG en 99 mujeres que solicitaron los servicios de AE.$^{37}$ En este estudio se logró establecer, de acuerdo con la probabilidad de embarazo establecida por Wilcox y colaboradores, ${ }^{38}$ la eficacia anticonceptiva del LNG administrado durante las fases preovulatoria y posovulatoria del ciclo ovárico. Los resultados demostraron la ausencia de embarazos en mujeres tratadas con LNG en la fase preovulatoria; sin embargo, el número de embarazos observados en mujeres tratadas en la fase posovulatoria no fue diferente respecto del número de los esperados (cuadro III). Estos resultados concuerdan con los obtenidos en otros estudios descritos con anterioridad en relación con el efecto preovulatorio del LNG como AE y contribuyen a descartar otros mecanismos de acción en la fase posovulatoria. Por otra parte, estas observaciones explican la eficacia (57-95\%), así como las fallas atribuibles al método (15\%).

\section{Conclusiones}

La AE de progestina sola (plan B) representa una opción para la prevención de embarazos no deseados. La eficacia del método no es comparable con la obtenida con los anticonceptivos hormonales combinados (98-100\%), por lo que no se recomienda su uso regular como método anticonceptivo. Su mecanismo de acción es todavía controvertido; no obstante, por la evidencia acumulada, es posible establecer sus efectos sobre la ovulación y sobre algunos procesos relacionados con la migración espermática en el tracto reproductivo femenino. Los estudios de eficacia anticonceptiva han permitido establecer que el intervalo entre el coito y el tratamiento representa una de las evidencias indirectas más importantes para descartar los efectos posovulatorios de este método. Estos datos se han confirmado en estudios con mejor diseño experimental dirigidos a establecer el mecanismo de acción anticonceptiva de la progestina sola. En términos generales, por la evidencia científica generada, incluidos los estudios estadísticos sobre la efectividad, así como los consensos derivados de las reuniones de expertos y las agencias internacionales como la Organización Mundial de la Salud (OMS), la Federación Internacional de Ginecología y Obstetricia (FIGO) y del Consorcio Internacional para Anticoncepción de Emergencia (ICEC), ${ }^{39,40}$ la AE de progestina sola ejerce sus efectos antes de la unión de los gametos (fecundación) y muy probablemente sólo al interferir con los mecanismos encargados de la función ovulatoria. Por otro lado, la

\section{Cuadro III}

Efectividad del leVonorgestrel administrado en LAS FASES PREOVULATORIA Y POSOVULATORIA Del CICLO OVÁRICO

\begin{tabular}{|c|c|c|c|}
\hline $\begin{array}{l}\text { Días del coito respecto } \\
\text { de la ovulación* }\end{array}$ & $\begin{array}{c}\text { No. de mujeres en riesgo } \\
\text { de embarazo }\end{array}$ & Administración de LNG & $\begin{array}{c}\text { No. de embarazos esperados/ } \\
\text { observados }\end{array}$ \\
\hline Días -5 a -2 & 34 & Preovulatoria & $4.15 / 0$ \\
\hline Días - I a 0 & 17 & Posovulatoria & $3.45 / 3$ \\
\hline Totales (-5 a 0) & 51 & Preposovulatoria & $7.60 / 3$ \\
\hline
\end{tabular}

*Día 0

Tomado de la referencia 37

LNG: levonorgestrel 
mayor parte de la información generada permite descartar razonablemente efectos posteriores a la fecundación, en particular los relacionados con los mecanismos de receptividad endometrial (implantación).

\section{Agradecimientos}

Parte de los estudios de esta revisión recibió el apoyo financiero del Consejo Nacional de Ciencia y Tecnología. La médica especialista Marta Durand es alumna del Programa de Doctorado en Ciencias Biomédicas, de la Facultad de Medicina de la Universidad Nacional Autónoma de México. Los autores agradecen a todos aquellos quienes brindaron su apoyo y asesoría para la elaboración de este artículo, en especial a la Dra. Patricia Uribe Zúñiga, Directora del Centro Nacional de Equidad de Género y Salud Reproductiva, de la Secretaría de Salud, y a la Dra. María del Carmen Cravioto Galindo, Investigadora del Departamento de Biología de la Reproducción, del Instituto Nacional de Ciencias Médicas y Nutrición Salvador Zubirán.

\section{Referencias}

I. Croxatto HB, Devoto L, Durand M, Ezcurra E, Larrea F, Nagle C, et al. Mechanism of action of hormonal preparations used for emergency contraception: a review of the literature. Contraception 200 I;63: I I I-I2I. 2. Glasier A. Emergency poscoital contraception. N Engl J Med 1997;337:1058-1064.

3. Trussell J, Raymond EG. Statistical evidence concerning the mechanism of action of the Yuzpe regimen of emergency contraceptive pills. Obstet Gynecol 1999;93:872-876.

4. Trussel J, Ellertson C, von Hertzen H, Bigrigg A, Webb A, Evans M, et al. Estimating the effectiveness of emergency contraceptive pills. Contraception 2003;67:259-265.

5. Durand M, Cravioto MC, Raymond EG, Durán-Sánchez O, CruzHinojosa ML, Castell-Rodriguez A, et al. On the mechanisms of action of short-term levonorgestrel administration in emergency contraception. Contraception 2001;64:227-234.

6. Croxatto HB, Ortiz ME, Muller AL. Mechanisms of action of emergency contraception. Steroids 2003;68:1095-1098.

7. Marions L, Hultenby K, Lindell I, Sun X, Stabi B, Gemzell Danielsson K. Emergency contraception with mifepristone and levonorgestrel: mechanism of action. Obstet Gynecol 2002;100:65-71.

8. Ling WY, Robichaud A, Zayid I, Wrixon W, MacLeod SC. Mode of action of dl-norgestrel and ethinylestradiol combination in postcoital contraception. Fertil Steril 1979;32:297-302.

9. Kubba AA, White JO, Guillebaud J, Elder MG. The biochemistry of human endometrium after two regimens of postcoital contraception: a dl-norgestrel/ethinylestradiol combination or danazol. Fertil Steril 1986;45:5I2-516.

10. Yuzpe AA, Thurlow HJ, Ramzy I, Leyshon JI. Post coital contraception a pilot study.J Reprod Med 1974;13:53-58.

II. Swahn ML,Westlund P, Johannisson E, Bygdeman M. Effect of postcoital contraceptive methods on the endometrium and the menstrual cycle. Acta Obstet Gynecol Scand 1996;75:738-744.
12. Taskin O, Brown RW,Young DC, Poindexter AN, Wiehle RD. High doses of oral contraceptives do not alter endometrial $\alpha \mid$ and $\alpha v \beta 3$ integrins in the late implantation window. Fertil Steril 1994;61:850-855. I3. Raymond EG, Lovely LP, Chen-Mok M, Seppälä M, Kurman RJ, Lessey BA. Effect of Yuzpe regimen of emergency contraception on markers of endometrial receptivity. Hum Reprod 2000;15:235I-2355.

14. Ling WY,Wrixon W,Acorn T, Wilson E, Collins J. Mode of action of dlnorgestrel and ethinylestradiol combination in postcoital contraception III. Effects of preovulatory administration following the luteinizing hormone surge on ovarian steroidogenesis. Fertil Steril 1983;40:631-636.

15. Yuzpe AA, Lancee WJ. Ethinylestradiol and dl-norgestrel as a postcoital contraceptive. Fertil Steril 1977;28:932-936.

16. Hapangama D, Glasier AF, Baird DT.The effects of peri-ovulatory administration of levonorgestrel on the menstrual cycle. Contraception 2001;63:123-129.

17. Marions L, Cekan SZ, Bygdeman M, Gemzell Danielsson K. Effect of emergency contraception with levonorgestrel or mifepristone on ovarian function. Contraception 2004;69:373-377.

18. Croxatto HB, Fuentealba B, Brache V, Salvatierra AM, Alvarez F, Massai $R$, et al. Effects of the Yuzpe regimen, given during the follicular phase, upon ovarian function. Contraception 2002;65: I2I-I 28.

19. Croxatto HB, Brache V, Pavez M, Cochon L, Forcelledo ML,Alvarez $\mathrm{F}$, et al. Pituitary-ovarian function following the standard levonorgestrel emergency contraceptive dose or a single $0.75 \mathrm{mg}$ dose given on the days preceding ovulation. Contraception 2004;70:442-450.

20. Yeung WS, Chiu PC, Wang CH, Yao YQ, Ho PC. The effects of levonorgestrel on various sperm functions. Contraception 2002;66:453-457. 21. Bahamondes L, Nascimento JAA, Munuce MJ, Fazano F, Faúndes A. The in vitro effect of levonorgestrel on the acrosome reaction of human spermatozoa from fertile men. Contraception 2003;68:55-59.

22. Nascimento JA, Seppälä M, Perdigão A, Espejo-Arce X, Munuce $M]$, Hautala $L$, et al. In vivo assessment of the human sperm acrosome reaction and the expression of glycodelin- $\mathrm{A}$ in human endometrium after levonorgestrel-emergency contraceptive pill administration. Human Reprod 2007;22:2190-2195.

23. Brache V, Faundes A, Johansson E, Alvarez F.Anovulation, inadequate luteal phase and poor sperm penetration in cervical mucus during prolonged use of Norplant ${ }^{\circledR}$ implants. Contraception 1985;3 I:26 I-273. 24. Croxatto HB, Diaz S, Salvatierra AM, Morales P, Ebensperger C, Brandeis A. Treatment with Norplant ${ }^{\circledR}$ subdermal implants inhibits sperm penetration through cervical mucus in vitro. Contraception 1987;36: 193-201.

25. Kesserü E, Garmendia F,Westphal N, Parada J.The hormonal and peripheral effects of $\mathrm{d}$-Norgestrel in postcoital contraception. Contraception 1974;10:41 I-424.

26. Croxatto HB. Gamete Transport. In:Adashi EY, Rock JA, Rosenwaks Z, ed. Reproductive Endocrinology, Surgery, and Technology. New York, USA: Lippincot-Raven, 1996:386-402.

27. Ling WY,Wrixon MD, Zayid I,Acorn T, Popat R, Wilson E. Mode of action of dl-norgestrel and ethinylestradiol combination in postcoital contraception II. Effect of postovulatory administration on ovarian function and endometrium. Fertil Steril 1983;39:292-297.

28. Durand M, Cravioto MC, Castell-Rodríguez A, Larrea F. Expresión endometrial de genes y proteínas reguladas por progesterona posterior a la administración aguda de levonorgestrel. Rev Invest Clin 2004;56:550. 29. Ugocsai G, Rozsa M, Ugocsai P. Scanning electron microscopic (SEM) changes of the endometrium in women taking high doses of levonorgestrel as emergency postcoital contraception. Contraception 2002;66:433-437.

30. Landgren BM, Johannisson E,Aedo AR, Kumar A, Yong-en S. The effect of levonorgestrel administered in large doses at different stages of the cycle on ovarian function and endometrial morphology. Contraception 1989;39:275-289. 
3I. Kahlenbom C, Stanford J, Larimore WL. Postfertilization effect of hormonal emergency contraception. Ann Pharmacother 2002;36:465-470. 32. Muller AL, Llados C, Croxatto HB. Postcoital treatment with levonorgestrel does not disrupt postfertilization events in the rat. Contraception 2003;67:415-419.

33. Ortiz ME, Ortiz RE, Fuentes M, Parraguez VH, Croxatto HB. Postcoital administration of levonorgestrel does not interfere with postfertilization events in the new-world monkey Cebus apella. Hum Reprod 2004; 19:1352-1356.

34.WHO Task Force on Postovulatory Methods of Fertility Regulation. Randomised controlled trial of levonorgestrel versus the Yuzpe regimen of combined oral contraceptives for emergency contraception. Lancet 1998;352:428-433.

35. Piaggio G, von Hertzen H, Grimes DA,Van Look PFA. On behalf of the Task Force on Postovulatory Methods of Fertility Regulation.Timing of emergency contraception with levonorgestrel or the Yuzpe regimen. Lancet 1999;353:721.
36. Wilcox AJ, Baird DD, Weinberg CR. Time of the implantation of the conceptus and loss of pregnancy. N Engl J Med 1999;340: 1796-1799. 37. Novikova N,Weisberg E, Stanczyk FZ, Croxatto HB, Fraser IS. Effectiveness of levonorgestrel emergency contraception given before or after ovulation- a pilot study. Contraception 2007;75: I I2-I I8.

38. Wilcox AJ, Weinberg CR, Baird DD. Timing of sexual intercourse in relation to ovulation: effects on the probability of conception, survival of the pregnancy, and sex of the baby. New Engl J Med 1995;333:| I I7-I52I. 39. WHO Fact sheet. Levonorgestrel for emergency contraception. Geneva:WHO, 2005.

40. international Consortium of Emergency Contraception/International Federation of Ginecology and Obstetrics. Statement on mechanism of action of Levonorgestrel-only emergency contraceptive pills. New York: ICEC/FIGO, 2008 